\title{
Liquid Metal Processing and Casting Foreword
}

Over the past three decades, considerable effort has been made by researchers to improve our knowledge of mechanisms and processes involved in the field of liquid metal processing and casting (LMPC). Liquid metal processing and casting, including solidification, represents a key stage in the development of many metallic materials, since it provides the final product with most of its intrinsic properties. In order to gather the industrial and scientific communities in the field of specialty metals' melting and refining, the series of "International Symposium on Liquid Metal Processing and Casting" was started in 1994. Now held alternatively every two years between Santa Fe, NM, and Nancy, France, LMPC conferences provide a unique forum for intensive discussion on advanced experimental and mathematical modeling research.

During all of these meetings, this subject area has attracted great interest, and the latest meeting was no exception, providing an excellent spectrum of presented work in a total of 58 papers. During LMPC2007, held in Nancy in September 2007, the presentations and ensuing discussions spanned a range of scales from the theoretical mathematical models to full-scale industrial investigations. The papers were distributed in six sessions on vacuum arc remelting (VAR), electroslag refining (ESR), steel processing, solidification, titanium processing, and process modeling and optimization.

Of these presentations, one quarter of the presenters were asked to submit journal articles to be reviewed and published in this special section of Metallurgical and Materials Transactions B, highlighting the key scientific and engineering advances in the field. As expected, this selection of articles shows an intense interest in the advanced modeling of the processes, most of them including results from industrial processing to be related to the model predictions. Classical computational fluid dynamics methods are applied in most simulation cases, ranging from remelting processes to vacuum oxygen decarburization, ladle metallurgy, or cold crucible melting. A few articles directly address the solidification process, from experimental and theoretical perspectives, as well as the use of electromagnetic fields and their interaction with the melt. Slag/metal reactions are also investigated.

A large number of the contributions present investigations of the physics and control of consumable electrode remelting processes (VAR and ESR). Experimental investigations of final product performance, full-scale industrial experiments, and integrated modeling are all covered within these articles, which combine to form an excellent review of the process detail and provide an exceptionally good overview of how models can be applied to industrial processes.

In summary, this collection of articles provides an overview of the state-of-the-art research into both the experimental and computational investigations of liquid metal processing and casting. The articles included here show that our scientific understanding of these processes is still far from complete.

Organizers:

P.D. Lee

Department of Materials

Imperial College London

London SW7 2BP, United Kingdom

E-mail: p.d.lee@imperial.ac.uk

A. Mitchell

Advanced Materials Laboratories

University of British Columbia

Vancouver, BC V6T 1Z4, Canada

A. Jardy and J.P. Bellot

Laboratoire de Science et Génie des Matériaux et de Métallurgie (UMR 7584),

Ecole des Mines

54042, Nancy Cedex, France 